\title{
New Techniques in Project Management
}

\author{
Cameron Fisher \\ Massachusetts Institute of Technology, Cambridge, USA \\ Email: cameronf@wharton.upenn.edu
}

Received 13 October 2014; revised 23 November 2014; accepted 5 December 2014

Copyright (C) 2014 by author and Scientific Research Publishing Inc.

This work is licensed under the Creative Commons Attribution International License (CC BY). http://creativecommons.org/licenses/by/4.0/

\section{(c) (i) Open Access}

\begin{abstract}
Managing multiple candidate initiatives and in-flight projects across business units is a big challenge for organizations. Tracking progress of projects while balancing demand against available resources to ensure successful completion is difficult. Increasingly, organizations deploy a Project Portfolio Management (PPM) system. These new PPM solutions simplify portfolio governance and strive to guide enterprise innovation. For maximum benefits, the frameworks that shepherd initiatives and bolster project efficiency must not obstruct ideation, bottom-up creativity and entrepreneurship.
\end{abstract}

\section{Keywords}

Project Management, Portfolio Optimization, Innovation

\section{Introduction}

Corporations seek to accelerate project development cycles to gain faster go-to-market execution. With new PPM and governance methods (e.g. resource management, demand management, internal and external sourcing algorithms, workload balancing), improved rigor and efficiency are attainable.

Controls for cost, scope, compliance and quality dimensions need to be managed to maximize healthy innovation. Nevertheless, care must be taken to not manage allocations so strictly that enterprise innovation is obstructed or frustrated.

To achieve balance, Project and Portfolio Management solutions facilitate an ideation pipeline and optimal project mix that considers risks, rewards, trends and technology tradeoffs. Strategic planning and tactical management featuring executive dashboards shepherd proposed initiatives, garner approvals, manage work, reduce unwelcome project "surprises" and bolster transitions to operational readiness and success.

Large and complex initiatives typically entail project lifecycle phases such as:
1) Define
2) Design
3) Develop
4) Unit test
5) Integrate 
5) System test

6) Deploy

7) Stabilize

8) Handover as project closes

9) Maintain

The legacy shortcomings of Waterfall project lifecycle management include:

- Tendency for scope creep

- Inflexibility after definition phase that rely upon rigid change controls

- Stakeholders and customers are less intimately involved

- Tangible results manifest later, after extensive fund commitment

- Project health monitoring and project performance tracking are influenced by behavioral factors

The shortcomings of Waterfall methods are well established. Increasingly, Rapid Application Development, Spiral and Agile approaches are prevalent. This holds true for information technology projects, non-IT projects and major initiatives that partially rely on information technology to succeed. For most industry sectors these days, relatively few new programs are devoid of a significant IT component.

Such variants from Waterfall promise to better control risk and decrease reliance on upfront documentation and to involve more prototyping to capture story points. Time to value can be fostered with compressed-duration development cycles and shorter-duration version releases. Often, teams prefer an Agile approach to "move fast" and not stall progress to documenting their work, especially when multiple tool sets are involved. The prototyping itself is pointed at, and represents a manifestation of progress and market readiness.

Furthermore, stakeholders participate actively throughout and help brainstorm requirements and interfaces and prioritizations. Iterations and releases are monitored as burn down unfolds and velocity metrics are monitored. The prototype itself becomes a reliable representation of progress earlier in the timelines and thereby helps manage risk.

However, if you are the CXO with limits on time and budget, how can you grapple with such activities and know where you stand? Executives must oversee many diverse projects that are under development or transitioning to operations.

Hence, PPM has arisen as a key framework for organizations to manage and coordinate initiatives.

\section{What Is PPM?}

What is PPM? Why would an enterprise embrace PPM? How is PPM achieved? What cautionary factors apply to ensure that innovation thrives, risks are mitigated and programs succeed?

\subsection{The Compelling Need for PPM}

What if there is a severe internal resource shortage for IOS programmers and Android programmers? Go to market timing is often vital, as firms rush to launch a wave of mobile and cloud applications that their consumers can embrace. If development for a project is rushed in an attempt to meet customer deadlines or incentive-driven performance targets, quality assurance could get swept under the rug, and bite back later. The cost of defective work and re-work will ultimately affect outcomes.

How would you plan multiple initiatives to avoid shortages or bottlenecks? What would be the means to diversify risk with multiple parallel projects concurrently in-flight? Striving to not being dependent on scarcity of suppliers, workers, technologies, platforms, channels, business representatives, executive leaders or other resource conflicts?

To manage portfolio demands and balance the ecosystems of resources, PPM can help! Nevertheless, PPM needs to be undertaken properly, by an experienced team of company representatives, stakeholders and professional implementers.

As we shall see, it's a delicate balancing act to effectively inject:

- Discipline

- Accountability

- Rigor

- Visibility

Taking care to not stifle:

- Creativity 
- Innovation

- Intrapreneurship

- Motivation

- Coordination

- Information sharing

\subsection{How to Approach PPM}

The best approach will require managers and executives to:

- Identify new and existing ideas for pipeline funnel

- Classify by project types: platform, derivative, R \& D, partnered, M + A, etc.

- Estimate the average time and resources needed for each project type, based on standards and past initiatives

- Identify current and attainable resource bandwidth

- Design a target mix of projects taking into consideration corporate Mission, Goals, Strategies, Risk Preferences and Critical Success Factors

- Balance demand for projects with resources on hand or attainable

- Choose projects based on weighted prioritization criteria

- Close doomed initiatives as early as possible

- Refine program and product development methods for repeatability and scalability

Senior executives and the CIO require dashboards to monitor the portfolios that consist of in-flight projects. These collections of projects progress through a lifecycle of ideation, setup, funding, development, deployment and stabilization.

The diagram shows a dashboard screenshot for a corporate portfolio. The horizontal axis is finish date and the vertical axis represents projects in the portfolio; a smaller red circle reflects a relatively less costly project which is performing poorly; a larger green circle reflects a larger budget and a favorable project health. As market conditions and an organization's activity unfolds, portfolio composition and priorities will shift.

The graphic illustrates a portfolio of benefits and budgeted costs for colored sponsor groups of a PPM solution user interface. Larger spheres reflect bigger budget initiatives. For example, blue is Finance, purple is IT and pink is customer service. Benefits can be on a projected pre-deployment basis or on a realized and audited post-deployment basis. Selection of report type and filters generates the business insight output. Additional dashboard graphics can display budgeted amounts by finish date. Green can represent on-schedule. Yellow can denote cautionary issues or alerts are flagged. Red can signify a problematic project status at risk of cancellation or spending freeze (Figure 1).

The early concepts of a central PMO (project management office) and CPM (critical path method) techniques flourished under corporate professional management and NASA space race of the 1960's.

The Project Management Institute certifies project management practitioners with a PMP designation. PPM systems are a relatively recent solution category in the discipline of project management. PPM should not be confused with ERP (Enterprise Resource Management) and ITSM (IT Service Management) perspectives. Albeit at times, their boundaries can overlap.

\subsection{Who Uses PPM?}

Headcount, skills inventory and resource availability dynamically adjust when overseeing a portfolio of in-flight projects. The balance of demand and supply will continue to adjust. The resource assigned for a project's phases hold an enlistment status that will progress through a cycle of: planned, "soft" reserved, readied/trained, assigned and released. The different roles that may negotiate these assignments can include:

- Stakeholder

- Business representative

- Partner/Supplier

- Supervisor

- Project manager

- Resource manager

- PMO

- Trainer

- etc. 


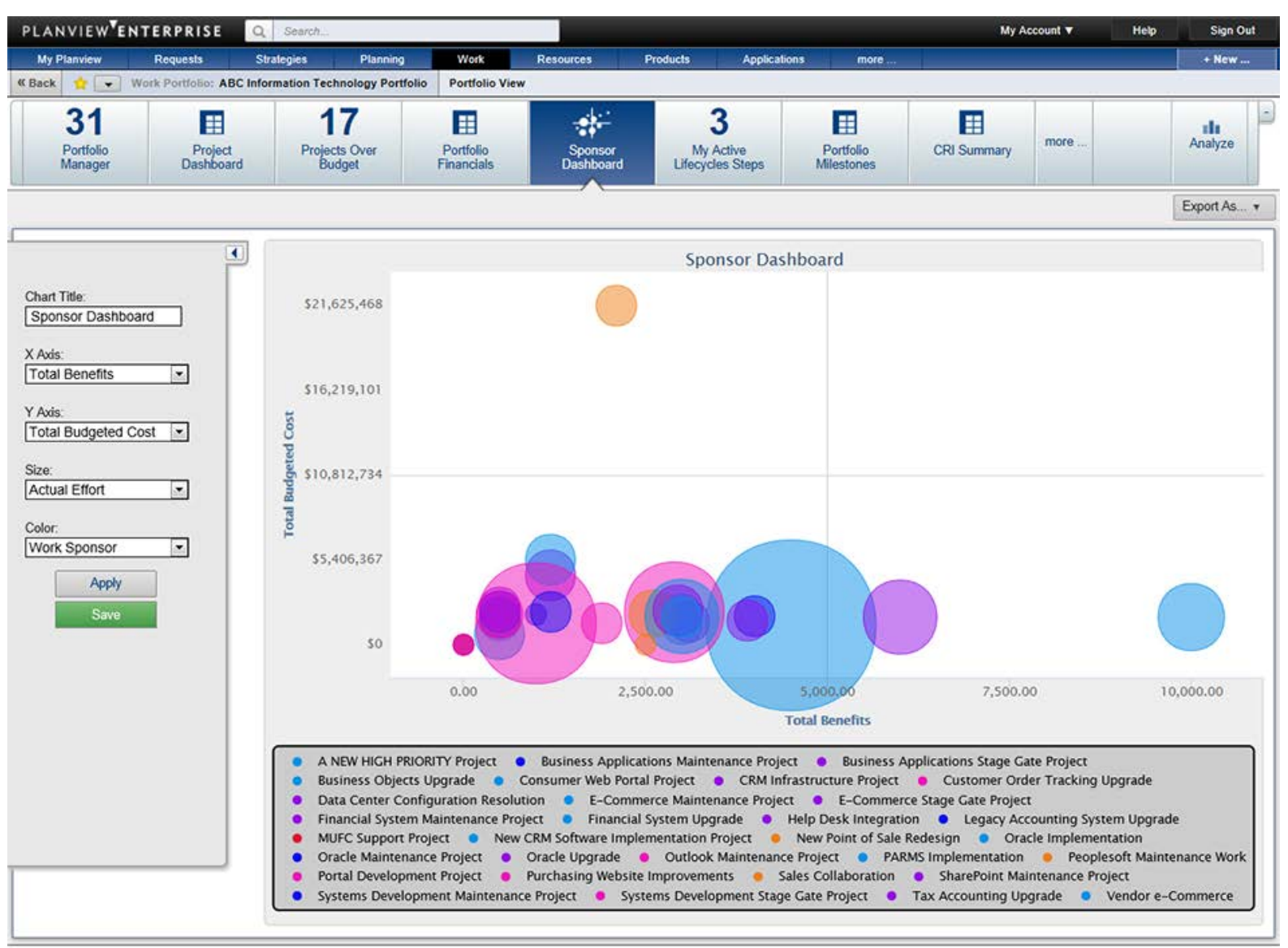

2013 Planview. Inc. All riahts reserved

Figure 1. Portfolio dashboard of in-flight projects by sponsor (C) Planview 2013 [1].

\subsection{Where Is PPM Procured?}

Accenture and other prominent system integrators obtain PPM from leading solution providers. For example, HP, CA and Planview are vendors that publish software licensing for deploying enterprise scale PPM solutions at complex organizations (e.g. Fortune 500 firms). The top providers and their implementation approaches are discussed later in this document.

\subsection{What Cautions Are Needed When Deploying PPM?}

Centralization of ideas should not be excessively top down such that grass-roots contributions are stifled. Adequate representation from across the ecosystem of participants (potential users, users, customers, employees, executives and other stakeholders) need to provide regular input.

\subsection{What Will PPM Not Do?}

Exclusions include enterprise resource planning systems, IT service management systems and general ledger systems. However, PPM must integrate and co-exist with key financial and human resource systems.

\subsection{Why Bother to Deploy PPM?}

PPM enables a CEO and PMO (program management office) to oversee a range of initiatives and project types.

Different project candidates exhibit different profiles and resource requirements across the portfolio. Operational Planning systems need to be complementary and be able to constructively co-exist. Investments and resource allocations should be guided by the best minds collectively but still accept candidates from a range of 
origins. Early warning alerts for troubled initiatives enable a project to be flagged and scrutinized. If necessary, a get well plan can help limit risks and remediate the concerns. Trouble prone projects or runaway projects are cancellable earlier with fewer surprises.

With improved governance, business process management and investment decisions, the organization can better manage: Money, Labor, Processes, Data and Deliverables across the firm. This enables the CXO and CIO to:

- Make effective IT portfolio decisions and resource allocations

- Compress duration for delivery of commitments

- Balance agility with governance

- Pursue strategic and tactical excellence

- Encourage innovation and discipline

- Coordinate multiple programs concurrently

\subsection{Fundamentals of PPM}

The following terms and phrases are used within Project Portfolio Management:

- Portfolio

- Project plan

- Demand management

- Resource management

- Budget

- Timesheet

- Project financials

- Project health

- Risks

- Issues

\subsection{PPM Application Providers}

Many organizations start their Project Portfolio Management information solution by purchasing an enterprise solution from a leading vendor such as:

- HP

- Planview

- Computer associates

- Microsoft

Some vendor offerings focus on information technology while others focus on financial or industry specializations. Furthermore, as Oracle and SAP expand their footprints, some overlap of boundaries will occur between PPM and enterprise resource planning (ERP).

Once configured, PPM solution modules help manage the intake of innovation idea proposals as well as resource allocations. Deliberately managing phases and gates can help coordinate resources and provide visibility (i.e. early warning of quality, manpower or financial problems).

\section{Corporate PPM Frameworks Can Invoke Phases and Gates}

How can you manage a range of diverse initiatives and project types? PPM enables strategic and effective handling to meet the demand for new projects while overseeing a myriad of resource types, skill sets and availabilities.

A typical framework for large organization will include:

- Concept to approval

- Define and design

- Specify and build

- Test

- Go live preparations

- Shake-out/Stabilization 
Phases and gates can exist and be overseen by PMO formally and systematically. However, with a mature PPM solution, the result is an end-to end system for a global enterprise which contains automated processes and the ability to filter personalized dashboard views. The result is a balanced portfolio of projects with more effective oversight (Figure 2).

A pipeline and funnel of projects evolve and gain formal sponsorship so that high potential-projects can attract resources (hardware, software, talent) and form a team as the enterprise develops a high performance culture. The project tasks are completed by teams comprised of on-demand workers assembled just in time. The high performance teams perform work, roll-on to a project and efficiently roll-off. Resources are released timely and promptly are eligible for re-deployment to other enterprise projects.

\section{Phases and Gates}

- PPM uses standards boards that can leverage PMI and PMBOK [2]

- PPM leverages proven methods to propose, approve, execute and report

- Waterfall is well-established due to link to standards and experiences

- Waterfall is becoming less entrenched as Agile gains proponents

In some cases, a project is problem-prone early. In a Waterfall approach, if not properly managed, the set of expenditures that projects attract can become a death spiral that gobbles up scarce resources in terms of talent, funding, timelines and missed market opportunity. Care should also be taken to ensure phases and gates do not impair democratizing idea generation and grass-roots innovation.

In recent years, project methods, have increasingly enlisted Agile approaches with iterative releases and more collaborative development approaches. The result is better intra- and inter-project communications that enable timely go-to-market launches. PPM systems have incorporated new work flows that accommodate Agile development efforts. PPM systems can insert a project structure template which accommodate to scrum meetings, story points, prototyping, prioritizing, velocity management and roll-up reporting [3].

Phases provide structure for realizing a defined body of work from ideation, setup, design, build, stabilization through closure. Phases reflect a progression of idea-candidate-program/project-close. Each phase has "go/no-go" gates in which steering committees and management review "idea screens" (i.e. candidate project proposals) that advance through the progression, are cancelled or put on hold.

Phase 1 -Concept to approval.

Phase 2-Business definition and design.

Phase 3-Technical design and build.

Examples of control gates for a phase in the concept to approval stream contain increasingly detailed specifications such as:
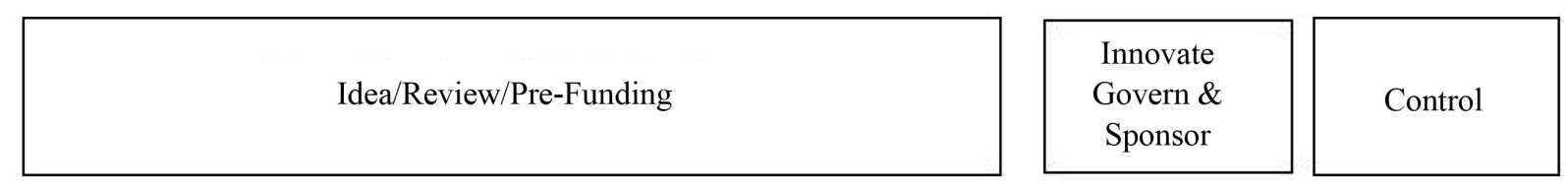

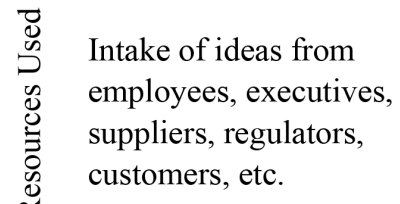
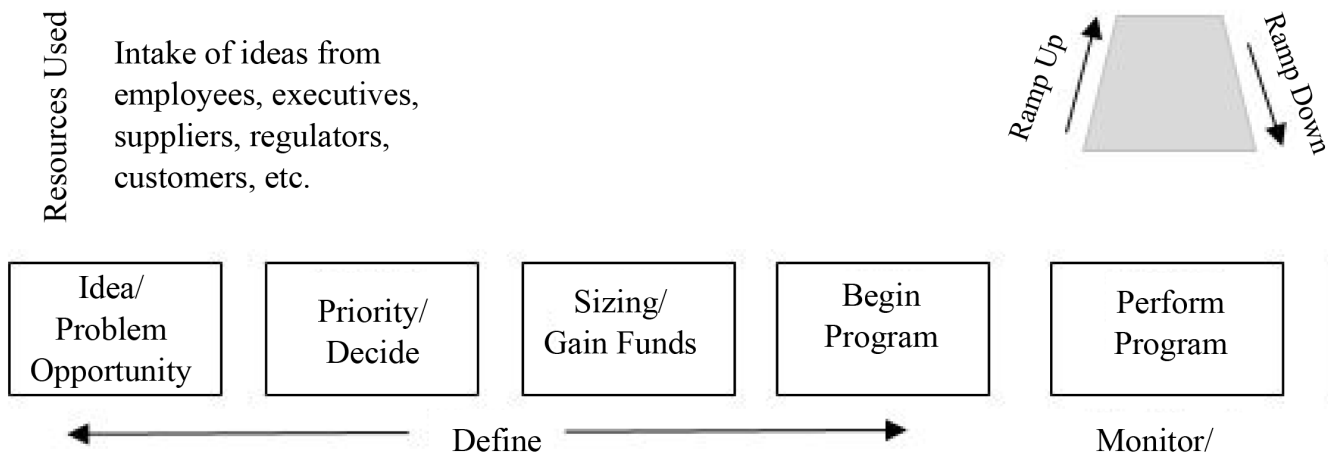

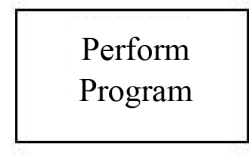

Monitor/ Improve
Transition to

Run

Operate \&

Sustain

Figure 2. Project lifecycle management framework. 
- Project proposal idea screen with value statement

- Initiative prioritization review

- Prioritization review meeting discussion

- Solution options and financials estimates

- Business case approval

- Resource staffing plan at initial high level

Examples of control gates for a phase in the business definition \& design stream contain increasingly detailed specifications (e.g. Formalized Portfolio Management) examine requests, programs and projects for solution architectures, solution designs and go-to-market plans.

The definition and design phases aim to clarify the business requirements detail; and to explain how technology and change management will address the business needs functionally and non-functionally. Agile techniques can also enrich the collection of stakeholder input, improve the developers' collaboration and accelerate solution releases.

Examples of control gates for a phase in the Technical Design \& Build stream contain increasingly detailed specifications such as:

- Detailed solution build

- Testing scripts

- User acceptance testing

- Updated project plan

- Project closure plan

Shortcomings of PPM can include:

- All desired information is not necessarily fully input into a PPM solution

- Designated managers who view system reports will not necessarily have full visibility into ranks

- Control and approvals need to fully enlist both centralized and decentralized approvers

- Unless intentionally circumvented via a slush fund, will turn a blind eye to (or compete with) less formal (or rogue/shadow/skunk work) systems since disparate spreadsheets lack audit ability, traceability and a transparent single source of truth

- Corporate acquisitions that are carefully and not hastily integrated can help overcome a resistance to change or "not invented here syndrome"

\section{Beware of Obstructing Key Innovations}

People at some firms are inclined to resist innovation-why? The status quo may be perceived as stable, secure, straightforward or less stressful [4]. And the capex/opex rationing and budgeting process can constrain ideations that progress through gates and are deliberately funded in phases or milestones. Proposals should align with changing market conditions, corporate mission, resources and viability. And competition, pricing, features, capacities and capabilities are also dynamic factors to weigh.

The source of an original idea or refinement may derive from a range of stakeholders and participants. It is important to realize that excessive gates or phases should not interfere with the incubation or intake of ideas from employees, executives, suppliers, regulators or customers.

A disruptive innovation can be competence-destroying to the status quo incumbents instead of competence creating; and provoke a shift to activities and techniques that the firm may lack current and future prowess. Incumbents could resist or sabotage ideas that seem threatening to an individual or group, even if an innovative ideas holds promise for long term organizational rewards.

"Innovation atrophy will set in unless creative people fight it. That's where the CIO has to set the tone.” [5] Information Week, May 27, 2011

Where a few number of users perceive a need, the lead users tend to innovate earliest. The role of users (not just suppliers) in advancing innovation is vital to recognize and leverage. Hence, a PPM ideation and requirements definition should accommodate employee bottom-up as well as customer-provided feedback.

\section{Demand Management and "Operations versus Innovation" Mix}

Demand Management can aid in weighing the trade-off of operations versus innovation. Strategic decision- 
making is easier when the more numerous and predictable tactical decisions are segregated out.

Historically, the CIO/CTO's expenditures were highly consumed by "keeping the show on the road". Consequently, innovation was often under-emphasized. In recent years however, advances occurred in IT manageability and affordability. For example, due to utility computing and Cloud capabilities, many firms are striving to "flip the equation" mix from $80 / 20$ to $50 / 50$ or potentially even $20 / 80$, depending on their industry and competitive climate.

\section{Balancing Tradeoff of "Keeping Show on the Road" versus Innovation}

Upon investigating the merits of PPM, the question may arise: What value can PPM deliver? Answer: Both tactical and strategy coordination, frameworks, lifecycle management, single-source-of-truth and resource allocation to name a few. In other words, a chess-board view of the internal and external resource landscape that encompasses:

- Planning

- Execution

- Tracking of projects

\section{Project Cycle Attributes and Demand Types}

A portfolio will encounter and handle a range of demand types. The cycle time and resources needed to accomplish a straightforward work request is short, with relatively few actors, and lower complexity. At the other end of the spectrum, are highly strategic projects with many contributors, many dependencies and many work streams. The complex and strategic efforts are usually longer term in duration from project inception to closure.

An example of highly defined and repeatable is say, a tactical maintenance request, or a de-commissioning. In contrast, a project with complexity, ambiguity, and extensive resources and scope will drive a need for more numerous highly talented workers and greater expenditures. In other words, a range exists in the types of projects handled (Figure 3).

The budget characteristics vary for such projects. The nature of the demand will dictate how and when resources are assigned; be they programs, projects, work orders or service requests. Depending upon the industry, the versatility and transferability of resources will vary in responding to programs, projects, releases and work requests.

A unified system will help coordinate actors/tasks/milestone deadlines/utilization, etc. The cross-pool interchange of resources can be accomplished. And, greater versatility of resources can be accomplished through cross-training and by recognizing the common tasks within distinct project structure models.

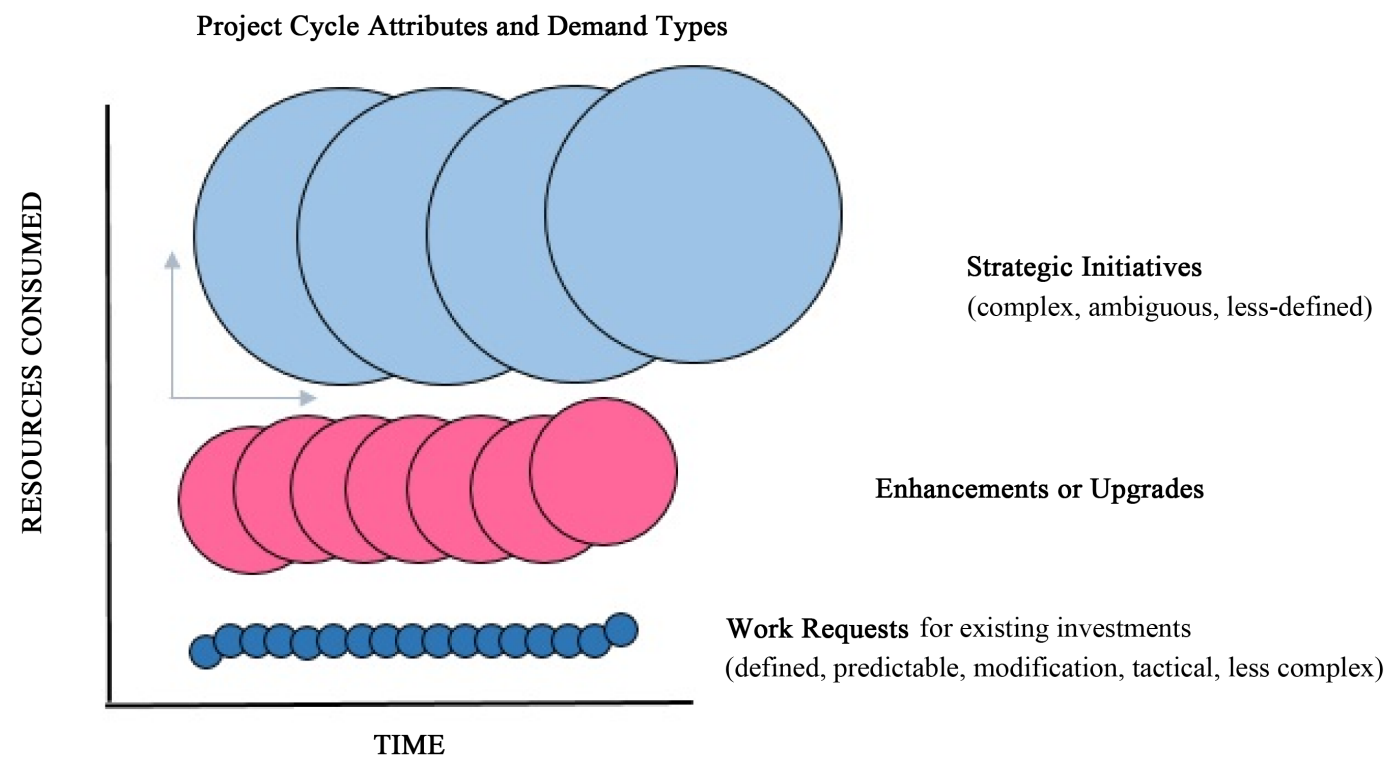

Figure 3. Project type characteristics. 


\section{Weighted Scoring}

Organizations strive to consume their resources to attain maximum value. Weightings of attributes are useful to produce a composite score to reflect projects' risk, urgency and business impact.

Multiple business units compete for scarce resources and capital is rationed after assessing:

- Market opportunity

- ROI, IRR, Payback (projected)

- Community or environmental impact

- Competitor advantage

- Technical complexity and breadth of impact and integrations (e.g. infrastructure, platform, applications)

- Program difficulty

- Labor relations

- Accessible skill sets

- Facilities and capacities

The scoring results are indicators that enable discussions about tradeoffs, decision-making and execution planning.

\section{Innovation and Dissemination}

“Innovation by users provides a very necessary complement to, and feedstock for manufacturer innovation.” [6] Democratizing Innovation, MIT Press

An impressive culture of innovation at Massachusetts General Hospital (MGH) where Dr. Nat Sims has been successful at attracting and nurturing ideas for medical innovation. Some people might question whether reliance on Dr. Sims represents a bottleneck and a less efficient method to funnel candidates for incubation as compared to an institutionalized PPM system.

Without PPM, can MGH really comprehend what "the right hand is doing and what the left hand is doing" on an enterprise scale?

What if an idea is not "exciting” or "cutting edge” but nonetheless indicate a high ROI? Do such ideas curry less favor and suffer in funding? Would a mundane idea for transforming building security ever see the light of day compared to breakthrough surgery?

In addition to Dr. Sims, particular comprehensive system solutions at MGH exist for tracking idea candidates from concept to approval. An on-site evangelist can influence and complement an institutionalized PPM system. A personal touch is not mutually exclusive to a rigorous info system that could manage portfolios of concepts/programs/projects that progress or dropout of the MGH pipelines toward payoff or rejection.

PPM system should take care to not create an autocracy of sources for innovation. Implemented correctly, the PPM system will enable a diverse source of employees, contractors, suppliers, partners, executives and virtual teams to ideate and shepherd candidates through a comprehensive selection and development process.

\section{Funnel Management}

Each new idea is a candidate for funding, refinement and fostering transformation. The intake funnel that projects progress through for PPM should not be so onerous that worthy candidates get frustrated, stalled or deflected.

The PPM will accommodate multiple acknowledge business units, but needs to be cautious not to involve an undue hierarchical bureaucratization. Any organization may have political fiefdoms arise, but PPM can help reduce favoritism, cronyism and not-invented-here biases. Constructed properly and governed properly, PPM can help produce fact-based approval decisions objectively and more timely. Projects stand on their merit as they progress though realistic phases and gates (Figure 4).

Exceptions, alerts, risks and issues garner appropriate levels of visibility and remediation within the PPM system. Reporting is effective, distributed and punctual. Of course, reporting will often be dependent on the quality of information that Project Managers are submitting in the tracking mechanisms. If negativity or frustration becomes excessive, and innovative thinking becomes punished, the PPM system will suffer. To maximize adoption and success, PPM systems should ensure adequate inputs are collected for user feedback and continuous process improvement. Examples include proper integrations with employee feedback systems, supplier 


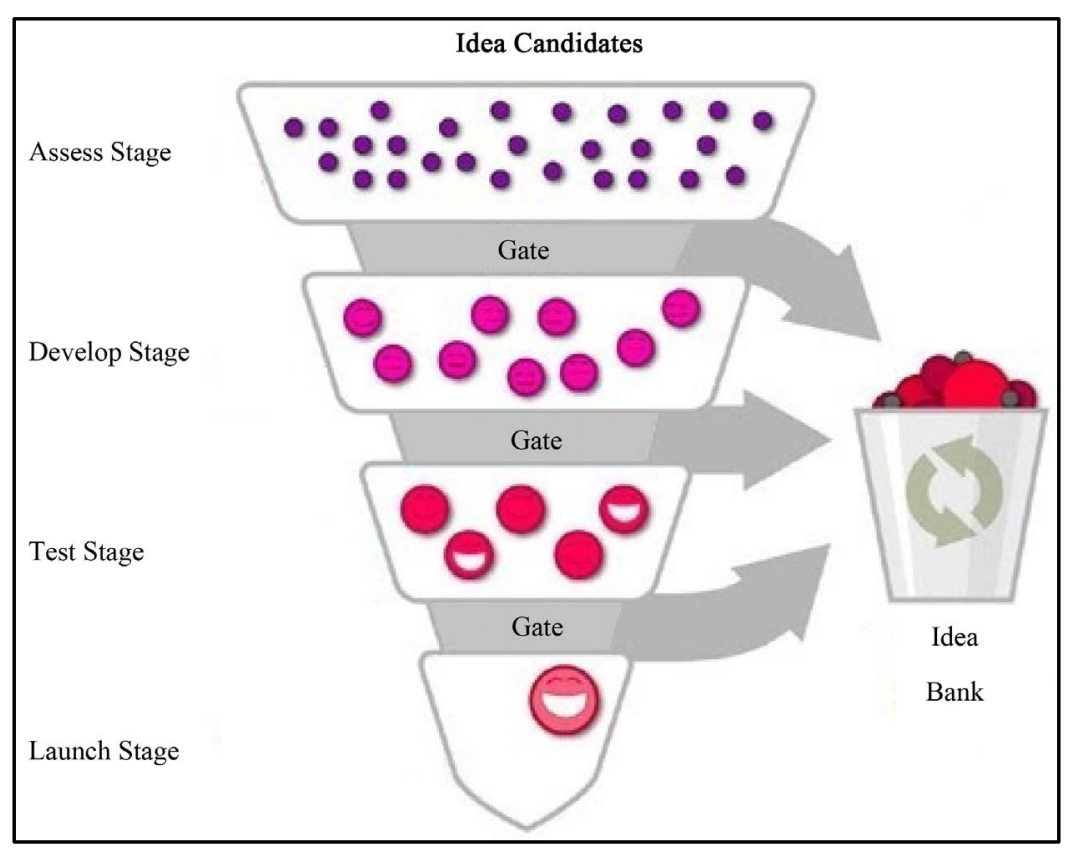

Figure 4. Ideas are candidates for project to be set up, to be killed, or to be banked as initiatives progress through gates.

feedback systems and customer feedback systems.

Avoiding a pitfall of reliance on centralized, top-down approvals is possible by including local representation of the review processes. Purse-strings are a chokepoint of control. If approvers are gatekeepers for a single funding source, the rejections could be viewed as stifling and discourage ideation.

Demand will exceed resources available to allocate and necessitate prioritization and phasing of dependencies and pre-requisites. Indeed, purse-strings are a chokepoint of control that roll-up to executive management. To avoid ivory towers, sustaining multiple "pockets" of funding can help reduce frustration and reduce out-of-touch impersonal relations among ideators, customers, approvers, users, project workers, etc.

While some less formal pockets of maverick actors may strive to avoid/circumvent the otherwise formalized tendency for "No" rejections, retaining their participation and enthusiasm by various means is helpful. Likewise, in the case of acquisitions, the "can-do" culture and nimbleness is important to retain. Otherwise, status quo play-it-safe tendencies will become inbred. The administrative system is built around the silo-ed and hierarchical bureaucracy. In contrast, a more socialized, Do-It-Yourself skunk works structure tends to offer grass-roots ideation close to the customer, and the promise of ongoing democratic contributions.

A range of actors typically hold an on-target bonus and incentives structured for diverse goals and personal motives. It is essential to unravel and rationalize the factors to align with agreed-upon mission and objectives. Behavioral distortions that attempt gaming the system, or "sandbagging" forecasts, or misleading overseers with misrepresented "earned value" calculations or other indictors should be investigated and dealt with to sustain PPM reporting integrity. A skilled PMO will adeptly navigate such factors.

\section{Rallying Non-Executives to Ideate}

Maintaining multiple funding sources can also permit multiple missions/strategies to co-exist for different business units or for different continents. In other words, encouraging more of an intra-firm marketplace than a strictly controlled central hierarchy is a healthy approach. There is a tradeoff of centralization and de-centralization that will differ by industry and participating firm.

At Intuitive Surgical in Silicon Valley, California the shop floor workers are regularly measured on the quantity and quality of their Employee Suggestions as a source for innovation. A range of successful new project initiatives were sparked from these employee ideas.

Although primarily from a manufacturing continuous process improvement standpoint, at a tactical shop-floor 
level, the leading edge processes and order-fulfillment occurs throughout the shift. The workers interact with end users for support issues, and often can anticipate user needs and use extensions. Each set of assembled finish products is tested and cleared for shipment (new) or remediation (existing) to handle user needs, the community and ecosystem creates suggestions for a range of improvements. This illustrates how innovation is a double edged sword. Innovation disrupts production efficiency-and production is a primary focus of many organizations.

End users are unique as compared to multi-level stakeholders, resellers or partners. End users are less feuding fiefdoms, and more goal-aligned. Users more willingly share innovative ideas as opposed to hoarding for commercial gain. Therefore, any PPM system should take care to solicit and elicit valuable innovation ideas from a range of stakeholders. Over-reliance on a bureaucratized PPM system would stifle the organization's propensity and capacity to innovate effectively.

An organization like MGH should foster use of less formal pockets for funding as well as a systematic enterprise-wide approach typical of large corporations when managing their PPM funnel. In all cases, security and compliance is overarching. Boards handling oversight and accountabilities should consider all funding approvals as important "gates". Ideas and projects not approved can be banked for future consideration and reactivation.

Of course, it is inappropriate to rely on disparate Excel spreadsheets across islands in the enterprise. The promulgation of state of the art, and necessary adherence is more rapidly and effectively disseminated with an enterprise-wide PPM system. Relying solely on a few champion charismatic/persuasive actors to usher which initiatives gain organizational support is short-sighted and in-bred. A comprehensive PPM system should accommodate the formal and less formal venues and vehicles of innovation, without being obstructive.

\section{Summary}

As discussed above, PPM helps overcome drawbacks of less formal systems. Namely, improvements are realized in ideation, setup, coordination, financial management, risk management and closure. Business units should not be silo-ed. Indeed some resources and techniques thrive best when a shared pool of workers are dispatchable to project teams.

PPM systems meet the need to adequately define responsibility for an organization-wide purview of funding candidates, investment diversification and funding allocations.

PPM systems will not unduly autocratize innovation—as long as outlets and encouragement can still occur. Employee suggestion plans, user forums, virtual user communities, user "hacks" and 360 degree customer surveys should still continue to spark key innovations, to manage and disseminate within enterprises.

For the example of MGH described earlier, a project portfolio system became better aligned with health pioneering motives. "Smart" drug infusion pumps greatly increased safety, effectiveness and efficiency of dispensing pharmaceuticals. A range of stakeholders could have erected resistance to innovativeness. Yet, particular physicians were willing to introduce disruptive change. Fortunately, the ability to shepherd funding and resources was not overly buttoned-up by a central PPM or a highly restrictive Phase-Gate system.

As explained, the relatively strict frameworks and timelines of Project Portfolio Management systems can impede bottom-up user creativity. Caution is worthwhile so that PPM regimes are not criticized for instances of being autocratic, bureaucratic, slow or stifling. To avoid such pitfalls, best practices should include:

- Enhanced communication flow between Technology Change agents and the business units serving the customers

- Centralized and standardized demand management process-supplemented by further features that nurture creativity and go beyond the status quo customer base

- New channels of input from employees, customers, suppliers and all stakeholders

- Visibility into the current and future resource pool

- Analytical insight into critical resources available to programs and projects

- Efficiency in projects' administration and oversight, including PMO efforts

- Balance assignable work (demand) with resources obtainable (time, people and money).

- Control over outsourced and off-shore development and services

Some professionals might complain that PPM fosters incremental (not breakthrough) improvements on the periphery. Or improvements are limited to the core existing customer base, careful to avoid substantial cannibalization of the status quo.

Knowing how and where to find lead users and opinion leaders is a ripe source of innovation. A large per- 
centage of the most successful start-ups are founded by lead users who are acting and experimenting close to their particular need. Yes, "Necessity breeds invention". But armed with PPM solutions and knowing common PPM pitfalls will help to:

- nurture creativity, innovation and entrepreneurship

- expand enterprise ranks that germinate ideation

- understand significance of correlation to parallel portfolios

- increase quality of project health oversight, risks and issues

- shepherd initiatives approved, cancelled, in-flight and completed

\section{References}

[1] Planview Inc. Product User Interface.

[2] (2000) A Guide to the Project Management Body of Knowledge. 2000 Edition, Project Management Institute, Newtown Square.

[3] Parker, D. and King, R. (2011) A Marriage Made in Heaven: Agile and Project Portfolio Management, Collabnet.

[4] Christenson, C. (2007) The Innovator's Dilemma: When New Technologies Cause Great Firms to Fail. Harvard Business Review Press, Cambridge.

[5] Murray, C. (2001) Innovation Atrophy: How Companies Can Fight It. Information Week.

[6] von Hippel, E. (2005) Democratizing Innovation. MIT Press, Cambridge. 
Scientific Research Publishing (SCIRP) is one of the largest Open Access journal publishers. It is currently publishing more than 200 open access, online, peer-reviewed journals covering a wide range of academic disciplines. SCIRP serves the worldwide academic communities and contributes to the progress and application of science with its publication.

Other selected journals from SCIRP are listed as below. Submit your manuscript to us via either submit@scirp.org or Online Submission Portal.
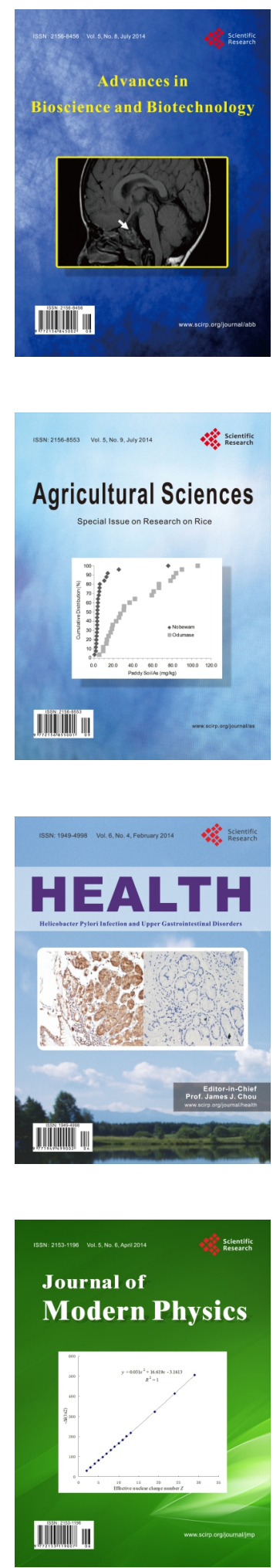
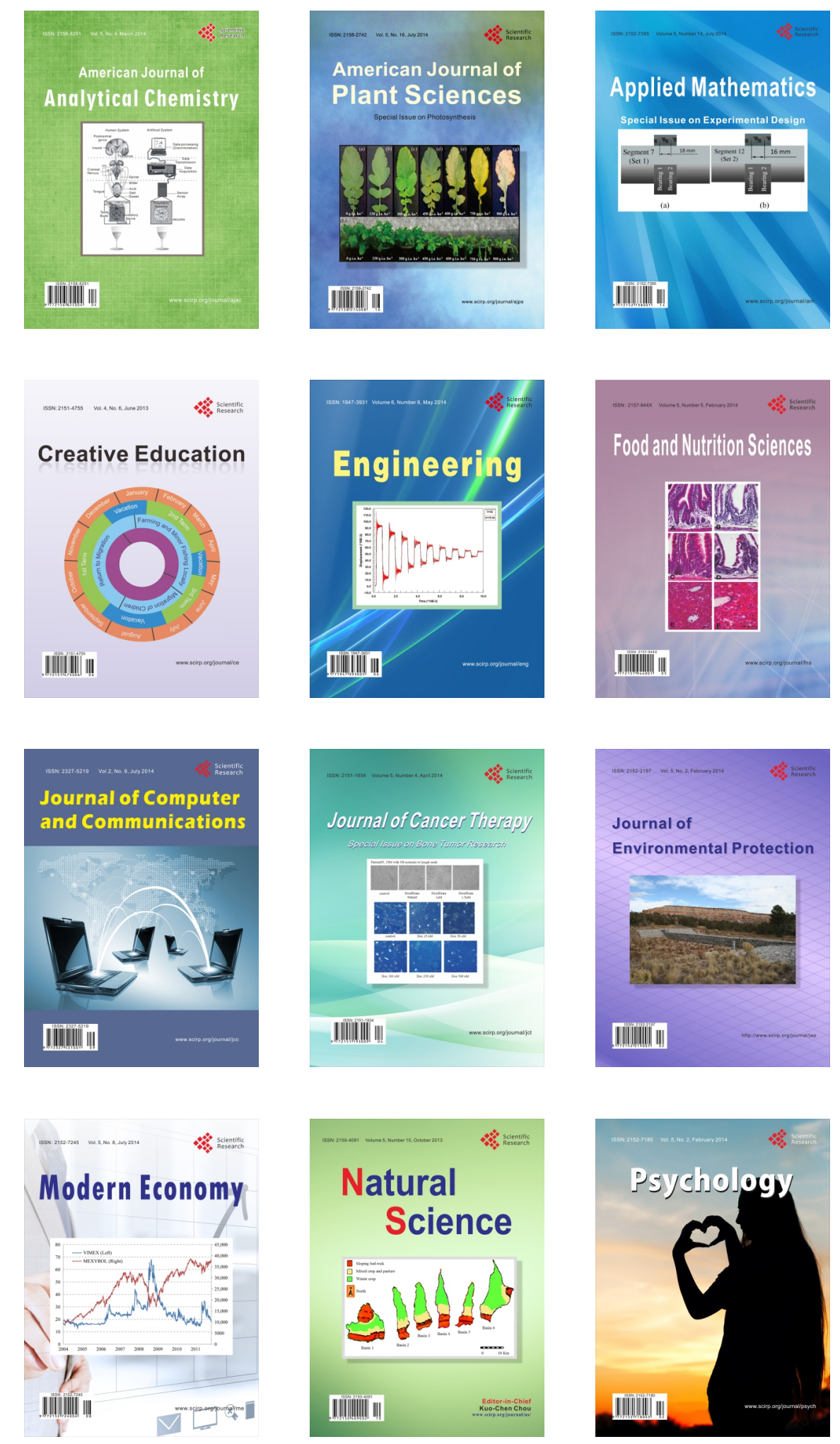